TRANSACTIONS OF THE

AMERICAN MATHEMATICAL SOCIETY

Volume 178, April 1973

\title{
KERNELS IN DIMENSION THEORY
}

\author{
BY
}

\section{J. M. AARTS AND T. NISHIURA $\left({ }^{1}\right)$}

ABSTRACT. All spaces are metrizable.

A conjecture of de Groot states that a weak inductive dimension theory beginning with the class of compact spaces will characterize those spaces which can be extended to a compact space by the adjunction of a set of dimension not exceeding $n$. Nagata has proposed a variant of this conjecture as a means of finding insights into the original conjecture. (See Internat. Sympos. on Extension Theory, Berlin, 1967, pp. 157-161.) The proposed variant replaces compact with $\sigma$-compact.

The present paper concerns a study of strong inductive dimension theory beginning with an arbitrary class of spaces. The study is motivated by the above two conjectures. It indicates that a theory of kernels is a more natural by-product of inductive theory than a theory of extensions. An example has resulted which, with the aid of the developed theory and the Baire category theorem, resolves the second conjecture in the negative. The original conjecture is still unresolved. It is also shown that the notion of kernels results in a further generalization of Lelek's form of the dimension lowering map theorem (Colloq. Math. 12 (1964), 221-227. MR 31\#716).

1. Introduction. At the outset, we agree that all spaces are metrizable.

The present paper is motivated by problems which relate extensions $Y$ of a space $X$ and the dimension of the remainder $Y \backslash X$. Let us state the general problem precisely. Let $\mathcal{P}$ be a topologically closed family of spaces and $n$ be a natural number. Then find a characterization of those spaces $X$ for which there is an extension $Y \in \mathcal{P}$ with $\operatorname{dim} Y \backslash X \leq n$. Classes of spaces which have been considered are the class of compact spaces [14] and the class of topologically complete spaces [1]. For these classes, characterizations have been found. As one might suspect, the characterizations involve dimension theoretic techniques.

There are three fundamental dimension theoretic techniques, weak inductive, strong inductive, and cover. The characterization in the compact case is given by covering techniques. The topologically complete case is handled by the strong inductive technique. It is a long standing conjecture of de Groot that the compact case has a weak inductive technique solution ([4], [5]). Nagata has proposed as a candidate for a class the $\sigma$-compact spaces as one in which a weak inductive

Received by the editors August 16, 1971 and, in revised form, March 20, 1972. 54E35.

AMS (MOS) subject classifications (1970). Primary 54F45, 54D40, 54D45; Secondary

Key words and phrases. Dimension theory, extensions, deficiency, kernel.

(1) Both authors were partially supported by the National Science Foundation Grants, NSF GP-12915 and NSF GP-28572. 
characterization could be found [12]. Such a characterization would perhaps shed some light on the conjecture of de Groot in the compact case.

With the two weak inductive conjectures and the strong inductive characterization mentioned above in mind, we develop in the present paper an abstract theory of strong inductive invariants. Our theory shows that, in classes such as $\sigma$-compact spaces, the notion of extension is not as natural as the notion of a kernel, i.e., subspaces $Y$ of $X$ such that $Y \in \mathcal{P}$. This discovery has led to a negative solution to Nagata's problem. A second consequence of the notion of kernel is a generalization of the dimension lowering map theorem of Hurewicz (see \$5.2). This generalization is sharper than a similar theorem of Lelek [8]. After an initial section $(\$ 2)$ of definitions, we devote $\$ \$ 3,4,5,7$ to an abstract development of the strong inductive theory and its relationship to kernels. $\$ 6$ is devoted to resolving Nagata's conjecture and finally $\$ 8$ is devoted to some theorems concerning extensions.

2. Preliminaries. This section is devoted to the notational conventions, basic definitions and statements of simple propositions which will be useful later.

2.1. Conventions. M will always be used to denote the class of metrizable spaces.

If $A$ is a subset of a space $X$, its closure and boundary will be denoted by $\bar{A}$ and $B(A)$, respectively. In the event that the space $X$ needs to be emphasized, we will use the notation $\mathrm{cl}_{X}(A)$ and $B_{X}(A)$, respectively.

A subclass $\mathcal{P}$ of $\mathbb{N}$ will be called topologically closed if $Y$ is a member of $\mathcal{P}$ whenever $Y$ is homeomorphic to a member of $\mathcal{P}$. Unless stated to the contrary, all classes considered in the paper are to be topologically closed. From $\$ 3$ on, all classes considered are to be nonempty. We shall use the words class and family interchangeably.

Ind and ind will denote the usual strong and weak inductive dimension [11].

2.2. Definition of $\mathcal{P}$-surplus and $\mathcal{P}$-deficiency. For each class $\mathcal{P}$ of spaces and each space $X$, we say a space $Y$ is a $\mathcal{P}$-kernel $(\mathcal{P} \cdot$ bull $)$ of $X$ if $Y \in \mathscr{P}$ and $Y \subset X(Y \supset X)$. By the strong $\mathcal{P}$-surplus of $X$ we mean the infimum of the set of numbers $\{$ Ind $X \backslash Y: Y$ is a $\mathcal{P}$-kernel of $X\}$. This infimum will be denoted by $\mathcal{P}_{\text {-Sur }} X$. By the strong $\mathcal{P}$-deficiency of $X$ we mean the infimum of the set numbers $\{$ Ind $Y \backslash X: Y$ is a $\mathcal{P}$-hull of $X\}$. This infimum will be denoted by $\mathscr{P}$-Def $X$.

We define in an analogous manner the weak $\mathcal{P}$-surplus and weak $\mathcal{P}$-deficiency using the weak inductive dimension ind. The corresponding symbols will be $\mathcal{P}_{\text {-sur and }} \mathcal{P}_{\text {-def. }}$.

Proposition. $\mathcal{P}$-Sur $X=\mathscr{P}_{\text {-sur }} X$ whenever $X$ is separable.

Example. Let $\mathcal{S}=\{X: X$ is $\sigma$-compact $\}$. Let $Z=B \times I^{n}$ where $I$ is the unit interval and $B$ is the space of irrational numbers. Then $\mathcal{S}$-Sur $Z=\mathcal{S}$-sur $Z=n$. 
This is proved as follows. If $K$ is any $\mathcal{S}$-kernel of $Z$, then the natural projection of $K$ into $B$ is $\sigma$-compact. Since $B$ is not $\sigma$-compact, there is a point $q \in B$ such that $\{q\} \times I^{n} \cap K=\varnothing$. So $Z \backslash K \supset\{q\} \times I^{n}$ and Ind $Z \backslash K=n$.

2.3. Definition of dimensions which neglect a family $\mathcal{P}$. Let $\mathcal{P}$ be a family of spaces. Following Lelek [8] we define strong (weak) inductive invariants, $\mathcal{P}_{\text {-Ind }}\left(\mathcal{P}_{\text {-ind }}\right)$, induced by $\mathcal{P}$ as follows:

If $X$ is a space, then

(1) $\mathcal{P}$-Ind $X=-1(\mathcal{P}$-ind $X=-1)$ if and only if $X \in \mathcal{P}$,

(2) for each integer $n \geq 0, \mathcal{P}$-Ind $X \leq n\left(\mathcal{P}_{\text {-ind }} X \leq n\right)$ provided that each nonempty closed subset (each point) of $X$ has arbitrarily small open neighborhoods $U$ such that its boundary $B(U)$ has $\mathcal{P}_{\text {-Ind }} B(U) \leq n-1\left(\mathcal{P}_{\text {-ind }} B(U) \leq n-1\right)$.

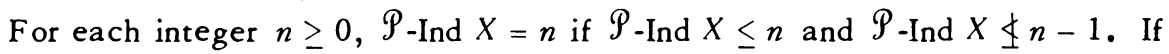
$\mathcal{P}$-Ind $X \Varangle n$ for each $n$, then $\mathcal{P}$-Ind $X=\infty$. Similarly, $\mathcal{P}_{\text {-ind }} X=n, n=0,1,2$, $\cdots, \infty$, are defined.

The requirement that the closed set be nonempty in condition (2) of the definition of $\mathcal{P}$-Ind $X$ is very important in order to cover the cases where $\varnothing \notin \mathcal{P}$. See the proof of Proposition 3 in $\$ 2.4$.

We will call the function $\mathcal{P}_{\text {-Ind }}(\mathcal{P}$-ind) defined on the class $\mathbb{M}$ of metrizable spaces the strong (weak) inductive dimension which neglects $\mathcal{P}$.

In the sequel $\mathcal{P}$-Ind $X$ and $\mathcal{P}$-ind $X$ will be computed for various spaces $X$ and classes $\mathcal{P}$. For more examples see [13].

In a manner analogous to $\mathcal{P}$-Ind, we next define a third type of dimension which neglects $\mathcal{P}$. This definition uses $\sigma$-locally finite bases, an important concept in dimension theory of metrizable spaces (see [11, Theorem II.2]). If $\mathcal{P}$ is a family of spaces then we define the basic dimension which neglects $\mathcal{P}, \mathcal{P}$-Bind, as follows:

If $X$ is a space, then

(1) $\mathcal{P}$-Bind $X=-1$ if and only if $X \in \mathcal{P}$,

(2) for each integer $n \geq 0, \mathcal{P}$-Bind $X \leq n$ if there exists a $\sigma$-locally finite open basis $\mathfrak{B}$ of $X$ such that for every $U \in \mathfrak{B}$ its boundary $B(U)$ has $\mathcal{P}$-Bind $B(U)$ $\leq n-1$.

$\mathcal{P}$-Bind $X=n, n=0,1, \cdots, \infty$, are defined as usual.

When $\mathscr{P}=\{\varnothing\}$, we will drop the prefixes of $\mathscr{P}_{\text {-Ind, }} \mathscr{P}_{\text {-ind }}$ and $\mathcal{P}_{\text {-Bind. It }}$. is well known that $B$ ind $=$ Ind $\geq$ ind.

2.4. Remarks and propositions. The study of inductive invariants chiefly aims at (1) generalizing dimension theory, (2) finding out what part of dimension theory is due to the inductive nature of the definition, and (3) discovering an interplay of the inductive invariants. These points will become evident as our development unfolds. 
Proposition 1. For any class $\mathcal{P}$ the functions $\mathcal{P}_{\text {-Sur, }} \mathcal{P}_{\text {-sur, }} \mathcal{P}_{\text {-Def, }} \mathcal{P}_{\text {-def, }}$ $\mathcal{P}_{\text {-Ind, }} \mathcal{P}_{\text {-ind }}$ and $\mathcal{P}$-Bind are topologically invariant.

Proposition 2. $\mathscr{P}_{\text {-ind }} \leq \mathscr{P}_{\text {-Ind, }} \mathscr{P}_{\text {-sur }} \leq \mathscr{P}_{\text {-Sur } \text { and }} \mathscr{P}_{\text {-def }} \leq \mathscr{P}_{\text {-Def. }}$

Example 1. Let $\mathcal{K}=\{X: X$ is compact $\}$. For the real line $R$ we have $\mathcal{K}$-Sur $R$ $=\mathcal{K}$-sur $R=\mathcal{K}$-Ind $R=1$ and K-def $R=\mathcal{K}$-Def $R=\mathcal{K}$-ind $R=\mathcal{K}$-Bind $R=0$.

$\mathcal{K}$-ind $X$ has been denoted by cmp $X$-the compactness degree of $X$-in earlier works ([4], [5]). The conjecture of de Groot [4] referred to in the introduction is the following:

Conjecture. $\mathcal{K}$-def $X \leq n$ if and only if $X$ is separable and cmp $X \leq n$.

The conjecture is true for $n \leq 0$. It remains open for all other $n$ [5].

Example 2. Let $\mathcal{C}=\{X: X$ is topologically complete $\}$. Then $\mathcal{C}$-Ind $X$ has been denoted by Icd $X$-the strong inductive completeness degree of $X$-in [1]. The following theorem is proved in [1].

Theorem. Icd $X \leq n$ if and only if C-Def $X \leq n$.

Example 3. Let $\mathcal{S}$ be as in the example in 2.2. Then $\mathcal{S}$-ind $X$ has been denoted by $\sigma$-cmp $X$ in [12]. It has been conjectured there that $\mathcal{S}$-def $X \leq n$ if and only if $X$ is separable and $\sigma-\operatorname{cmp} X \leq n$. We will prove this conjecture false in $\$ 6$ below.

Proposition 3. (1) If $\mathcal{P}$ and $\mathcal{Q}$ are two families with $\mathcal{P} \subset \mathcal{Q}$ then $\mathcal{P}$-Ind $\geq$

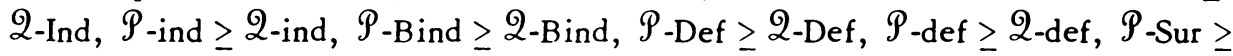
2-Sur and $\mathcal{P}$-sur $\geq 2$-sur.

(2) $\varnothing$-ind $X=$ ind $X+1, \varnothing$-Ind $X=$ Ind $X+1=\varnothing$-Bind $X$.

(3) $\varnothing \in \mathcal{P}$ if and only if $\mathcal{P}$-Ind $\leq$ Ind if and only if $\mathcal{P}$-ind $\leq$ ind if and only if $\mathcal{P}$-Bind $\leq$ Ind.

(4) If $\varnothing \notin \mathcal{P}$, then $\mathcal{P}$-Ind $X=0$ if and only if $X=\varnothing$.

Proof. We shall only indicate a proof of (4). For more details see [2] and

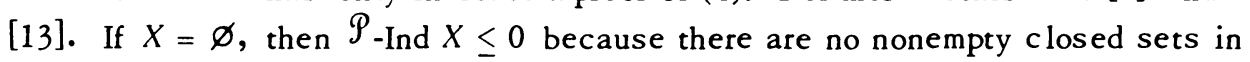
$\varnothing$. Since $\varnothing \notin \mathscr{P}, \mathcal{P}$-Ind $X=0$. If $X \neq \varnothing$, then, since $X$ is both open and closed, we must have $\varnothing \in \mathcal{P}$ in case $\mathcal{P}$-Ind $X \leq 0$.

Since the case $\mathcal{P}=\varnothing$ is completely determined, we will in the remaining sections always assume $\mathscr{P} \neq \varnothing$. This, of course, is not the same as $\varnothing \in \mathcal{P}$.

Finally, we remark that ideas related to inductive invariants have occurred in the literature prior to the works cited above. We refer the reader to [7, p. 187], and $[16, \mathrm{p} .96]$.

3. Properties of $\mathcal{P}$-Bind. In $\S 2$ we have already observed the relationship between strong and weak inductive dimensions which neglect $\mathcal{P}$. In this section we discuss the relationship of these two dimensions to $\mathcal{P}$-Bind. 


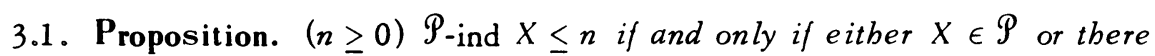
exists an open basis $\mathfrak{B}$ of $X$ such that $\mathcal{P}_{\text {-ind }} B(U) \leq n-1$ for every $U \in \mathfrak{B}$.

Theorem. $\mathcal{P}_{\text {-ind }} \leq \mathcal{P}_{\text {-Bind }}$.

The proof is a straightforward induction on $\mathcal{P}$-Bind $X$.

3.2. Definition of a framework. In many proofs it will be convenient to introduce the following notion of framework.

The pair $\left(\left\{U_{\gamma}: \gamma \in \Gamma\right\},\left\{F_{\gamma}: \gamma \in \Gamma\right\}\right)$ is called a framework of a $\sigma$-locally finite bas is of $X$ if

(1) $\left\{U_{\gamma}: \gamma \in \Gamma\right\}\left(\left\{F_{\gamma}: \gamma \in \Gamma\right\}\right)$ is an open (closed) collection;

(2) $\varnothing \neq F_{\gamma} \subset U_{\gamma}$ for every $\gamma \in \Gamma$;

(3) if $\left\{V_{\gamma}: \gamma \in \Gamma\right\}$ is an open collection such that $F_{\gamma} \subset V_{\gamma} \subset U_{\gamma}, \gamma \in \Gamma$, then it is a $\sigma$-locally finite basis of $X$.

Proposition. In every space there exists a framework of a $\sigma$-locally finite basis.

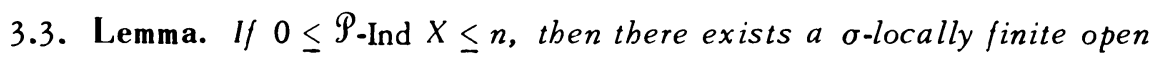
basis $\mathfrak{B}$ of $X$ such that $\mathcal{P}$-Ind $B(\bar{V}) \leq n-1$ for every $V \in \mathfrak{B}$.

The lemma easily follows from the definitions of a framework (observe that $\varnothing \neq F_{\gamma}$ in (2)) and of $0 \leq \mathscr{P}_{\text {-Ind }} X \leq n$.

From this lemma we obtain by induction on $\mathcal{P}$-Ind $X$ the following theorem.

Theorem. $\mathscr{P}$-Bind $\leq \mathscr{P}_{\text {-Ind }}$.

3.4. Theorem. If $X$ is separable, then $\mathcal{P}_{\text {-ind }} X=\mathcal{P}_{\text {- }}$ ind $X$.

Proof. In view of the theorem of $\$ 3.1$ we need only prove $\mathcal{P}$-Bind $X \leq \mathcal{P}_{\text {-ind }} X$. This holds for $\mathcal{P}_{\text {-ind }} X=-1$. The induction on $\mathcal{P}_{\text {-ind }} X$ is the same as in [11, pp. 90-91 ], where we must delete the empty set from the constructed basis $B$ when $\mathcal{P}_{\text {-ind }} X=0$, since $\varnothing$ need not be a member of $\mathcal{P}$.

3.5. Proposition. Let $\left\{B_{\gamma}: \gamma \in \Gamma\right\}$ be a $\sigma$-locally finite closed collection of subsets of a space $X$. Let, for each $\gamma \in \Gamma,\left\{B_{\gamma}^{\delta}: \delta \in \Delta_{\gamma}\right\}$ be a $\sigma$-locally finite (closed) collection of the subspace $B_{\gamma}$. Then $\left\{B_{\gamma}^{\delta}: \delta \in \Delta_{\gamma}, \gamma \in \Gamma\right\}$ is a $\sigma$-locally finite (closed) collection of subsets of $X$.

Theorem. Let $\mathcal{P}$-Bind $X \leq n$. Then there exists a $\sigma$-locally finite closed collection $\mathcal{C}$ of subsets of $X$ such that

(1) $\mathcal{C} \subset \mathcal{P}$,

(2) Ind $(x \backslash \cup \mathcal{C}) \leq n$.

Proof. If $\mathcal{P}$-Bind $X=-1$, then let $\mathcal{C}=\{X\}$.

Let $n \geq 0$ and assume the theorem true whenever $\mathcal{P}$-Bind $X \leq n-1$. Let $X$ 
be any space with $\mathcal{P}$-Bind $X=n$. Then there is a $\sigma$-locally finite open basis $\mathbb{B}$ of $X$ such that $U \in \mathfrak{B}$ implies $\mathscr{P}_{\text {- }}$ ind $B(U) \leq n-1$. By virtue of the induction hypothesis for each such $\dot{B}(U)$ there is a $\sigma$-locally finite closed collection $\mathcal{C}_{U}$ of subsets of $B(U)$ satisfying (1) $\mathcal{C}_{U} \subset \mathcal{P}$ and (2) Ind $\left(B(U) \backslash \cup \mathcal{C}_{U}\right) \leq n-1$. The above proposition gives $\mathcal{C}=\bigcup\left\{\mathcal{C}_{U}: U \in \mathfrak{B}\right\}$ is a $\sigma$-locally finite closed collection. Since $B(U) \backslash \cup \mathcal{C}$ is closed in $X \backslash \bigcup \mathcal{C}$, the sum theorem [11, Theorem II.1] gives Ind $(\bigcup\{B(U): U \in \mathfrak{B}\} \backslash \bigcup \mathcal{C}) \leq n-1$.

Also, Ind $(X \backslash \bigcup\{B(U): U \in \Re\}) \leq 0$. Hence by [11, II.1.C], we have Ind $(X \backslash \cup \mathcal{C}) \leq n$.

4. Monotone conditions. We devote this section to those theorems which result under some monotone conditions on $\mathcal{P}$.

4.1. Definition. A family $\mathcal{P}$ is said to be monotone if $Y \subset X \in \mathcal{P}$ implies $Y \in \mathcal{P}$. In the event that the implication holds only for closed subspaces $Y$ of $X$ then we say $\mathcal{P}$ is c-monotone. Similar concepts result for $F_{\sigma}, G_{\delta}$ and open subspaces $Y$ of $X$. We will prefix monotone with the symbols $F_{\sigma}, G_{\delta}$ and $o$ in the respective cases.

An extended real valued function $f$ on $M$ is said to be monotone if $f(X) \leq$ $f(Y)$ whenever $X \subset Y$. c-monotone, $F_{\sigma}$-monotone, $G_{\delta}$-monotone and o-monotone are similarly defined.

4.2. Theorem 1. A family $\mathcal{P}$ is monotone if and only if $\mathscr{P}$-Bind is monotone. Corresponding statements bold for closed, $F_{\sigma}$ and $G_{\delta}$-monotone. Analogous statements bold for $\mathcal{P}$-ind.

The proof of this theorem is a straightforward induction (cf. [13, Theorem 3.3]).

Remark. If $\mathcal{P}$ is $c$-monotone, then the proposition of $\$ 3.1$ is greatly simplified ("either $X \in \mathcal{P}$ or" can be dropped).

Theorem 2. A family $\mathcal{P}$ is c-monotone if and only if $\mathcal{P}$-Ind is c-monotone.

4.3. Proposition. $(n \geq 0)$ Suppose $\varnothing \in \mathcal{P}$. Let $A$ be a subset of $X$ with $0 \leq \mathcal{P}_{\text {-Ind }} A \leq n$. Then for any dis joint closed sets $F$ and $G$ of $X$ there exists an open set $U$ such that $F \subset U \subset X \backslash G$ and $\mathcal{P}_{\text {-Ind }}(B(U) \cap A) \leq n-1$.

In case $\mathcal{P}$ is $c$-monotone, the conditions $0 \leq \mathcal{P}_{\text {-Ind }} A$ and $\varnothing \in \mathcal{P}$ can be dropped.

Proof. There are open sets $V$ and $W$ satisfying $F \subset V, G \subset W$ and $\bar{V} \cap \bar{W}=$ $\varnothing$. Because $0 \leq \mathcal{P}$-Ind $A \leq n$ and $\varnothing \in \mathcal{P}$, there exists an open subset $D$ of $A$ satisfying $\bar{V} \cap \bar{A} \subset D \subset A \backslash \bar{W}$ and $\mathcal{P}_{\text {-Ind }} B_{A}(D) \leq n-1$.

Neither of the disjoint sets $F \cup D$ and $G \cup(A \backslash \bar{D})$ contains a cluster point of the other. Hence there is an open set $U$ such that $F \cup D \subset U \subset X \backslash \overline{B_{A}(D)}$ and $\bar{U} \cap(G \cup(A \backslash \bar{D}))=\varnothing$. A simple calculation yields $B_{A}(D)=B(U) \cap A$. 
If $\mathcal{P}$ is $c$-monotone, then $B(U) \cap A \in \mathcal{P}$ if $\mathcal{P}$-Ind $A=-1$ and $\varnothing \in \mathcal{P}$ (recall, we have agreed that $\mathcal{P} \neq \varnothing$ ).

4.4. Theorem. Suppose $\mathcal{P}$ is c-monotone. Let $X=A \cup B$ with $\mathcal{P}$-Ind $A \leq n$ and Ind $B \leq 0$. Then $\mathcal{P}_{\text {-Ind }} X \leq n+1$.

Proof. Let $F$ and $G$ be any disjoint closed sets in $X$. By Proposition 4.3 there exists an open set $U$ satisfying $F \subset U \subset X \backslash G$ and Ind $B(U) \cap B=-1$. Hence $B(U) \subset A$ which implies $\mathcal{P}_{\text {-Ind }} B(U) \leq n$ in view of Theorem 2 in $\$ 4.2$.

Corollary. Suppose $\mathcal{P}$ is c-monotone. Let $X=A \cup B$ with $\mathcal{P}$-Bind $A \leq n$ and Ind $B \leq 0$. Then $\mathcal{P}$-Bind $X \leq n+1$.

4.5. Theorem. Suppose $\mathcal{P}$ is c-monotone. Then $\mathcal{P}$-Ind $\leq \mathcal{P}$-Sur.

Proof. The theorem obviously holds for $\mathcal{P}$-Sur $X=-1$. Assume the theorem holds for $X$ with $\mathcal{P}_{\text {-Sur }} X \leq n-1$. Suppose $\mathcal{P}_{\text {-Sur }} X \leq n$. Let $Y$ be a $\mathcal{P}_{\text {-kernel }}$ with Ind $(X \backslash Y) \leq n$. By virtue of Proposition 4.3, for any disjoint closed subsets of $X$, there exists an open set $U$ such that $F \subset U \subset X \backslash G$ and Ind $(B(U) \cap(X \backslash Y))$ $\leq n-1$. Since $\mathcal{P}$ is $c$-monotone, $Y \cap B(U)$ is a $\mathcal{P}_{\text {-kernel }}$ of $B(U)$. Thus $\mathcal{P}_{\text {-Sur }} B(U) \leq n-1$. So, by the induction hypothesis, $\mathscr{P}_{\text {-Ind }} B(U) \leq n-1$. That is, $\mathcal{P}$-Ind $X \leq n$.

4.6. Theorem. Suppose $\mathcal{P}$ is c-monotone. Then $\mathcal{P}$-Bind $\leq \mathcal{P}$-Def.

Proof. The theorem is obviously true for $\mathcal{P}$-Def $X=-1$. Assume the theorem holds for all $X$ with $\mathcal{P}$-Def $X \leq n-1$. Suppose $\mathcal{P}$-Def $X \leq n$. That is, there is a

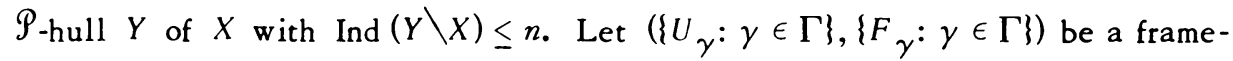
work of a $\sigma$-locally finite basis of $Y$. For every $\gamma \in \Gamma$, an open set $V_{\gamma}$ is selected such that Ind $\left(B_{Y}\left(V_{\gamma}\right) \cap(Y \backslash X)\right) \leq n-1$. Since $B_{Y}\left(V_{\gamma}\right)$ is a $\mathcal{P}_{\text {-hull of }}$ $B_{Y}\left(V_{\gamma}\right) \cap X$, the induction hypothes is gives $\mathcal{P}_{\text {-Bind }}\left(B_{Y}\left(V_{\gamma}\right) \cap X\right) \leq n-1$. Now $B_{X}\left(V_{\gamma} \cap X\right)$ is closed in $B_{Y}\left(V_{\gamma}\right) \cap X$. The c-monotonicity of $\mathcal{P}$ implies $\mathcal{P}_{\text {-Bind }} B_{X}\left(V_{\gamma} \cap X\right) \leq n-1 .\left\{V_{\gamma} \cap X: \gamma \in \Gamma\right\}$ is the required base.

4.7. Theorem. Suppose $\mathcal{P}$ is c-monotone and o-monotone. Then $\mathcal{P}$-Ind $\leq$ P-Def.

Proof. The proof is similar to that of the preceding theorem. Observe that, if $F$ and $G$ are disjoint closed subsets of $X$ and if $Z$ is any $\mathcal{P}_{\text {-hull of } X \text {, then }}$ $Y=Z \backslash\left(\mathrm{cl}_{Z} F \cap \mathrm{cl}_{Z} G\right)$ is another $\mathcal{P}_{\text {-hull }} X$ in which $F$ and $G$ have disjoint closures. Obviously, Ind $Y \backslash X \leq$ Ind $Z \backslash X$.

Remark. As follows from Example 1 in $\$ 2.4$ the condition that $\mathcal{P}$ be o-monotone cannot be dropped.

4.8. Theorem. (1) $\mathcal{P}$-Sur and $\mathcal{P}$-sur are monotone if and only if $\mathcal{P}$ is monotone. The corresponding statements bold for closed, $F_{\sigma}, G_{\delta}$ and o-monotone. 
(2) If $\mathcal{P}$ is c-monotone, then $\mathcal{P}$-Def and $\mathcal{P}_{\text {-def }}$ are c-monotone. The corresponding statements bold for $F_{\sigma}, G_{\delta}$ and o-monotone.

(3) If $\mathcal{P}$ is c-monotone, then $\mathcal{P}$-def $X=\mathcal{P}$-Def $X$ whenever $X$ is separable.

The easy proofs are omitted.

5. Sum theorems. In this section we shall develop sum theorems which are analogues of the sum theorem of dimension theory. Besides the condition of $c$ monotone, we necessarily will have to assume a sum closure for the class $\mathcal{P}$. We formalize these two conditions on $\mathcal{P}$ as $\mathrm{S} 1$ and $\mathrm{S} 2$ :

S1. $\mathcal{P}$ is c-monotone.

S2. $\mathcal{P}$ is closed for taking of unions of locally countable closed collections from $\mathcal{P}$.

It is a simple computation to show $\mathrm{S} 1$ and $\mathrm{S} 2$ are equivalent to $\mathrm{S} 1$ and

$\mathrm{S}^{\prime} 2 . \mathcal{P}$ is closed for taking of unions of $\sigma$-locally finite closed collections from $\mathcal{P}$.

A family which satisfies $\mathrm{S} 1$ and $\mathrm{S} 2$ is related to normal families of Hurewicz [6] and Morita [10]. Normal families require $\mathcal{P}$ to be monotone as well as S2.

Under the conditions of S1 and S2 we will establish the equivalence of the notions of $\mathscr{P}_{\text {-Ind and }} \mathcal{P}$-Sur. Using $\mathcal{P}$-Sur we shall prove the sum theorem and, in $\$ 7$, the decomposition theorem for $\mathcal{P}_{\text {-Ind }}$. Thus, the importance of $\mathcal{P}_{\text {-kernels }}$ for strong inductive invariants becomes evident (see Theorem 5.1).

5.1. The excision lemma. Suppose $\mathcal{P}$ satisfies condition $\mathrm{S}^{\prime} 2$. If $\mathcal{P}$-Bind $X$ $\leq n$, then there exists an $F_{\sigma} \mathcal{P}$-kernel $Y$ of $X$ with Ind $(X \backslash Y) \leq n$.

Proof. Suppose $\mathcal{P}$-Bind $X \leq n$. By virtue of the Theorem in 3.5 there exists a $\sigma$-locally finite closed collection $\mathcal{C}$ of subsets of $X$ such that Ind $(X \backslash \bigcup \mathcal{C}) \leq n$ and $\mathcal{C} \subset \mathcal{P}$. Clearly, $\cup \mathcal{C}$ is $F_{\sigma}$. Condition $S^{\prime} 2$ gives $\cup \mathcal{C} \in \mathcal{P}$.

Theorem. Suppose $\mathcal{P}$ satisfies conditions S1 and S2. Then $\mathcal{P}$-Bind $=\mathscr{P}$-Ind $=\mathscr{P}_{\text {-Sur. }}$. Furthermore each $X$ has an $F_{\sigma} \mathcal{P}$ kernel $Y$ with Ind $(X \backslash Y)=\mathcal{P}$-Sur $X$.

Proof. Theorems 3.3 and 4.5 give $\mathcal{P}_{\text {-Bind }} \leq \mathcal{P}_{\text {-Ind }} \leq \mathcal{P}_{\text {-Sur. The excision }}$

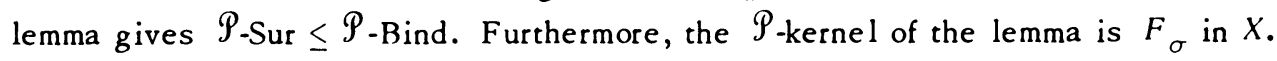

Corollary 1. Suppose $\mathcal{P}$ satisfies conditions S1 and S2. Then $\mathcal{P}_{\text {-Ind }} X \leq n$ if and only if $X=\bigcup\left\{X_{i}: i=0,1, \ldots, n\right\}$ for some $n+1$ subsets $X_{i}$ with $\mathcal{P}$-Ind $X_{0} \leq 0$ and Ind $X_{i} \leq 0, i=1, \cdots, n$.

From The orem 4.8 (1) we obtain

Corollary 2. Suppose $\mathcal{P}$ satisfies S1 and S2. Then $\mathcal{P}$-Ind is o-monotone.

Example 1. In Corollary 1 , Ind $X_{i} \leq 0, i=1, \cdots, n$, cannot be replaced by 
$\mathcal{P}_{\text {-Ind }} X_{i} \leq 0$. The sufficiency fails as the family $\mathscr{D}=\{X$ : Ind $X \leq 0\}$ shows. Consider the three-dimensional Euclidean space $X$. Then $\mathscr{D}$-Ind $X=2$. Since $X$ is the union of two subsets of dimension one, $X$ is the union of two subsets with D-Ind $\leq 0$.

Example 2. Let $\mathfrak{Q}(1)$ be the family of absolute Borel sets of additive class 1 ; that is, the family of $\sigma$-locally compact spaces [15]. Then $\mathbb{Q}(1)$ satisfies conditions S1 and S2. This follows easily since locally compact subsets of a space $X$ are $F_{\sigma}$ sets in $X$.

Example 3. Let $\mathcal{C}$ be the class of topologically complete spaces (Example 2 in $\$ 2.4)$. $C$ does not satisfy condition $\mathrm{S} 2$. We shall exhibit a space $X$ with $\mathcal{C}$-Ind $X<\mathcal{C}$-Sur $X$. Let $X=I \times Q \cup Q \times I$, where $I=\{t: 0 \leq t \leq 1\}$ and $Q=$ $\{t: t \in I$ and $t$ is rational\}. ( $X$ is regarded as a subspace of $I \times I$.) Since $X$ is of the first Baire category, $X \notin \mathcal{C}$. That is, $\mathcal{C}_{\text {-Ind }} X \geq 0$.

In view of the theorem mentioned in Example 2, $\$ 2.4$, we have $\mathcal{C}$-Ind $X=$ C-Def $X \leq$ Ind $(I \times I \backslash X)=0$. Hence $C_{\text {-Ind }} X=0$.

In view of $\$ 2.4$, Proposition 3, we have C-Sur $X \leq\{\varnothing\}$ - Sur $X=\operatorname{Ind} X=1$. Since each dense subspace of a space of the first Baire category is again of the first Baire category, every $C_{\text {-kernel }} X$ is not dense in $X$. Hence $\mathcal{C}_{\text {-Sur }} X \geq 1$ because Ind $U=1$ for every nonempty open subset $U$ of $X$.

5.2. Definition. An extended real valued function on the class $M$ of metrizable spaces is said to satisfy the sum condition if, for each $X, f(X) \leq$ $\sup \left\{f\left(E_{\gamma}\right): \gamma \in \Gamma\right\}$ whenever $\left\{E_{\gamma}: \gamma \in \Gamma\right\}$ is a nonempty locally countable closed covering of $X$.

Proposition. If $\mathcal{P}$-Sur satisfies the sum condition and $\varnothing \in \mathcal{P}$, then $\mathcal{P}$ satisfies condition S2. The converse bolds if $\mathcal{P}$ also satisfies the condition S1.

Proof. Let $\mathcal{C}=\left\{E_{\gamma}: \gamma \in \Gamma\right\}$ be a locally countable closed cover of $X$ and $\mathcal{C} \subset \mathcal{P}$. Either $\mathcal{C}=\varnothing$ or $\mathcal{C} \neq \varnothing$. If $\mathcal{C}=\varnothing$, then $\cup \mathcal{C}=\varnothing \in \mathcal{P}$. Suppose $\mathcal{C} \neq \varnothing$. Then $-1 \leq \mathcal{P}$-Sur $\cup \mathcal{C} \leq \sup \left\{\mathcal{P}\right.$-Sur $\left.E_{\gamma}: \gamma \in \Gamma\right\} \leq-1$. Hence $X=\bigcup \mathcal{C} \in \mathcal{P}$. Thereby the first statement is proved.

Suppose $\mathcal{P}$ satisfies S1 and S2. $\varnothing \in \mathcal{P}$ in view of S1. Let $\left\{E_{\gamma}: \gamma \in \Gamma\right\}$ be a nonempty locally countable closed covering of $X$. By virtue of the theorem in $\$ 5.1$, for each $\gamma \in \Gamma$ there is an $F_{\sigma} \mathscr{P}_{\text {-kernel } G_{\gamma}}$ of $E_{\gamma}$ such that Ind $E_{\gamma} \backslash G_{\gamma}=$ $\mathcal{P}_{\text {-Sur }} E_{\gamma}$. For each $\gamma \in \Gamma$ the set $G_{\gamma}=\bigcup\left\{G_{\gamma}^{i}: i=1,2, \ldots\right\}$ where each $G_{\gamma}^{i}$ is closed in $X$. By condition S1 we have $G_{\gamma}^{i} \in \mathcal{P}, \gamma \in \Gamma, i=1,2, \ldots$. Since $\left\{G_{\gamma}^{i}: \gamma \in \Gamma, i=1,2, \ldots\right\}$ is a locally countable closed collection we have $G=$ $\mathcal{U}\left\{G_{\gamma}^{i}: y \in \Gamma, i=1,2, \ldots\right\} \in \mathcal{P}$ by virtue of condition $\mathrm{S} 2$. Now the sum theorem of dimension theory gives Ind $\bigcup\left\{E_{\gamma} \backslash G: \gamma \in \Gamma\right\} \leq \sup \left\{\right.$ Ind $\left.\left(E_{\gamma} \backslash G\right): \gamma \in \Gamma\right\} \leq$ $\sup \left\{\right.$ Ind $\left.E_{\gamma} \backslash G_{\gamma}: \gamma \in \Gamma\right\}=\sup \left\{\mathcal{P}_{\text {-Sur }} E_{\gamma}: \gamma \in \Gamma\right\}$. Consequently $\mathscr{P}_{\text {-Sur }} X \leq$ $\sup \left\{\mathcal{P}_{\text {-Sur }} E_{\gamma}: \gamma \in \Gamma\right\}$. 
The proposition above is the sum theorem for $\mathscr{P}$-Sur. Combined with Theorem 5.1 this sum theorem gives an easy proof of the following theorem, which has already been proved in a more complicated way [2].

Theorem 1. The sum theorem for $\mathcal{P}$-Ind. Suppose $\mathcal{P}$ satisfies conditions S1 and S2. Let $\left\{F_{\gamma}: \gamma \in \Gamma\right\}$ be a locally countable closed covering of a space $X$ sucb that $\mathfrak{P}_{\text {-Ind }} F_{y} \leq n$ for $\gamma \in \Gamma$. Then $\mathfrak{P}_{\text {-Ind }} X \leq n$, i.e., $\mathfrak{P}_{\text {-Ind }}$ satisfies the sum condition.

Example. Let $£$ be the class of all spaces $X$ with the property:

Ind $X=\sup \{$ Ind $C: C$ is a compact subset of $X\}$.

This property was first discussed by Lelek [8]. The class $\mathcal{L}$ does not satisfy condition S1. Nonetheless, it can be proved that $\mathcal{Q}$-Sur satisfies the sum condition and in view of the proposition above, $\mathcal{Q}$ satisfies the condition $\$ 2$. The orems 3.5 and 3.3 now result in $\mathcal{L}$-Sur $\leq \mathfrak{Q}$-Bind $\leq \mathcal{L}$-Ind. Since $\mathcal{Q} \mathfrak{Q}(1)$ (see $\$ 5.1$, Example 2) we have $\mathbb{Q}(1)$-Ind $\geq \mathcal{Q}$-Ind.

The following theorem is an extension of Hurewicz's dimension lowering map the orem.

Theorem 2. Let $f: X \rightarrow f(X)$ be an arbitrary continuous map. Then Ind $X \leq$ Ind $f(X)+$ Ind $f+\mathscr{Q}$-Sur $X+1$.

Proof. Let $Y$ be an $\mathcal{L}$-kernel of $X$ such that Ind $X \backslash Y=\mathcal{L}$-Sur $X$. Then Ind $X \leq$ Ind $Y+\mathcal{L}$-Sur $X+1$. For each compact subset $C$ of $Y$ we have Ind $C \leq$ Ind $f(X)+$ Ind $f$ by Hurewicz's theorem.

Because of Theorem 3.4, we have for separable $X$, $\mathcal{E}$-Sur $X \leq \mathcal{L}$-Bind $X=$ $\mathcal{L}$-ind $X$. Consequently, the above theorem is an improvement of the corresponding theorem of Lelek [8], proved for separable $X$, where $\mathcal{L}$-Sur $X$ is replaced by $\mathcal{L}$-ind $X$. To see that $\mathcal{Q}$-Sur $\neq \mathcal{L}$-Bind, consider a separable space $X=X_{1} \cup X_{2}$, where $X_{1}$ and $X_{2}$ are disjoint closed sets, $X_{1}$ is compact, Ind $X_{1}=1, X_{2}$ is totally imperfect and Ind $X_{2}=2$. (A space is totally imperfect if each compact subset is countable.) Then $\mathcal{L}$-Sur $X=0$ since $X_{2}$ is the union of a one-dimensional subset and a zero-dimensional subset. Also, $\mathcal{L}$-ind $X=1$. This follows easily from the fact that ind $Y-1=\mathcal{Q}$-ind $Y$ for separable totally imperfect spaces $Y$ with ind $Y \geq 0$.

5.3. $S_{\sigma}$-spaces. In this section we show that the inductive invariant analogue of a theorem of Morita [10] remains true under our conditions S1 and S2.

A space $X$ is called an $S_{\sigma}$-space if $X$ is the countable union of closed subspaces with the star-finite property.

Theorem. Let $\mathcal{P}$ satisfy conditions $\mathrm{S} 1$ and $\mathrm{S} 2$. If $X$ is an $S_{\sigma}$ space, then $\mathcal{P}_{\text {-ind }} X=\mathcal{P}_{\text {-Ind }} X$. 
Proof. $X$ is the countable union of closed subspaces $X_{i}$ with the star-finite property. Hence, to prove $\mathscr{P}_{\text {-ind }} X \geq \mathcal{P}_{\text {Ind }} X$ it is enough to prove $\mathscr{P}_{\text {-ind }} Y \geq$ $\mathcal{P}_{\text {-Ind }} Y$ for each space $Y$ with the star-finite property, because $\mathcal{P}_{\text {-ind }}$ is c-mono-

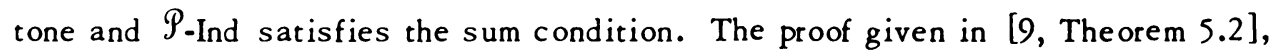
for spaces $Y$ with the star-finite property, that ind $Y \geq$ Ind $Y$, uses only the $c$ monotone property of ind and Ind and the sum property of Ind. Consequently, the very same proof can be used in the present situation.

6. $\sigma$-compactness deficiency. In this section we give a negative solution to the problem posed by Nagata $(\$ 2.4$, Example 3$)$. Let $\mathcal{S}$ denote the class of $\sigma$-compact spaces (see the example in $\$ 2.2$ and Example 3 in $\$ 2.4$ ). Let $Q(1)$ denote the class of $\sigma$-locally compact spaces (see Example 2 in $\$ 5.1$ ). Since $\mathcal{S} \subset \mathbb{Q}(1)$, by Proposition 3 in $\$ 2.4$ we have $\mathcal{S}$-Ind $\geq \mathbb{Q}(1)$-Ind, $\ldots, \mathcal{S}$-sur $\geq \mathbb{Q}(1)$-sur for the seven functions defined in $\$ \$ 2.2$ and 2.3 .

Since every $\sigma$-locally compact separable space is also $\sigma$-compact we have for each separable space $X$ : $\mathcal{S}$-Ind $X=\mathfrak{Q}(1)$-Ind $X, \ldots, \mathcal{S}$-sur $X=\mathfrak{Q}(1)$-sur for the seven functions.

From the proposition in $\$ 2.2$, Theorem 3.3 and Theorem 5.1 , it follows that for every separable space $X$ : $\mathcal{S}$-Ind $X=\mathcal{S}$-Bind $X=\mathcal{S}$-ind $X=\mathcal{S}$-Sur $X=\mathcal{S}$-sur $X$. In view of Theorem 4.8 for every separable space $X$ we have $\delta$-Def $X=\delta$-def $X$. Then, by virtue of Theorem 4.7 we have $\mathcal{S}$-Ind $X \leq \mathcal{S}$-def $X$ for every separable space $X$. We shall show that this inequality can be a strict inequality for some separable space $X$. Thus we give a negative solution to Nagata's problem.

6.1. Example. Here we present an example of a separable space $X$ with $\mathcal{S}$-Ind $X=0$ and $\mathcal{S}$-def $X=1$.

Let $I$ denote the unit interval, $Q=\{t: t \in I$ and $t$ is rational $\}$ and $B=I \backslash Q$. $X$ is the subspace of $I \times I \times I$ given by $X=(I \times I \times Q) \cup(B \times B \times B)$.

Since $I \times I \times Q^{\circ}$ is $\sigma$-compact we have $\mathcal{S}$-Ind $X \leq 0$ by Theorem 4.4 (there is also a direct proof for $\mathcal{S}$-ind $X \leq 0$ ).

$\mathcal{S}$-def $X \leq$ ind $(I \times I \times I) \backslash X=1$. We shall show $\mathcal{S}$-def $X \geq 1$. That is, if $Y$ is an $\delta$-hull of $X$, then ind $(Y \backslash X) \geq 1$. We may assume $X$ is dense in $Y$. Observe that $B \times B \times B$ is an absolute $G_{\delta}$ (i.e., a topologically complete space) which is dense in $Y$. Recall that a space which contains a dense subset of the second Baire category is a space of the second Baire category. Since $Y$ is a countable union of compact subsets, there is a compact subset $Z$ of $Y$ with nonvoid interior. So, $Z \cap X$ contains a topological copy of $X$, say $W$, which is closed in $X$. Since ind $Y \backslash X \geq$ ind $Z \backslash X \geq$ ind $\left(\mathrm{cl}_{Z} W\right) \backslash W$, we need only prove ind $\left(\mathrm{cl}_{Z} W\right) \backslash W \geq 1$. This will follow if we prove the compactness deficiency of $W$ exceeds 0 , that is $K$-def $W \geq 1$ (see Example $l$ in $\$ 2.4$ ). In view of Theorem 4.8 we need only find a closed subset $T$ of $X$ with $K$-def $T \geq 1$, since $K$ is $c$-monotone. Let $T=$ 
$\{0\} \times I \times Q . T$ is closed in $X$ and $\mathcal{K}$-def $T \geq 1$. The equality $\mathcal{K}$-def $T=1$ is proved in [5]. A more direct proof of $K$-def $T \geq 1$ can be obtained from the equality K-ind $T=K$-Bind $T$ (Theorem 3.4), the inequality $K$-Bind $T \leq K$-Def $T$ (Theorem 4.6), and the equality $K$-def $T=K$-Def $T$, provided $K$-ind $T=1$. To prove $K$-ind $T$ $=1$ we need only prove $K$-ind $I \times Q=1$. Let $f: I \times Q \rightarrow Q$ be the natural projection. For every sufficiently small open neighborhood $U$ of $(0,0) \in I \times Q, f(B)$ is a neighborhood of 0 in $Q$, where $B$ is the boundary of $U$. Hence $B$ is not compact. That is, $1 \leq \mathcal{K}$-ind $I \times Q$. Because $\mathcal{K}$-ind $I \times Q \leq$ Ind $I \times Q=1$, we have K-ind $I \times Q=1$.

Observe that $\delta$-def $X \geq 1$ implies $\delta$-ind $X>-1$. Consequently, $0 \leq \mathcal{S}$-ind $X \leq$ $\mathcal{S}_{\text {-Ind }} X \leq 0<1 \leq \mathcal{S}_{\text {-def }} X \leq 1$, and the example has the required properties.

7. The decomposition theorem. We have already commented in Example 1 of $\$ 5.1$ that a decomposition theorem for $\mathcal{P}$-Ind cannot hold in general. We will show

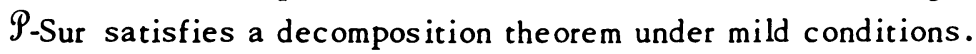

7.1. Proposition. If $\mathcal{P}_{\text {-Sur }} X \leq n$, then $X=\bigcup\left\{X_{i}: i=0,1, \ldots, n\right\}$ such that $\mathcal{P}_{\text {-Sur }} X_{i} \leq 0, \quad i=0, \cdots, n$.

This proposition follows immediately from the definition of $\mathcal{P}_{\text {-kernel }}$ and $\mathcal{P}$-Sur.

7.2. Definition. A family $\mathcal{P}$ is said to be additive if $X=A \cup B, A \in \mathscr{P}$ and $B \in \mathscr{P}$ imply $X \in \mathcal{P}$.

Proposition. $\mathcal{P}$ is additive if and only if $\mathcal{P}$-Sur $(A \cup B) \leq \mathcal{P}_{\text {-Sur }} A+\mathcal{P}_{\text {-Sur }} B+$ 1 for any subsets $A$ and $B$ of any space $X$.

Corollary 1. Let $\mathcal{P}$ be an additive family. Then $\mathcal{P}$-Sur $X \leq n$ if and only if $X=\bigcup\left\{X_{i}: i=0, \cdots, n\right\}$ with $\mathcal{P}_{\text {-Sur }} X_{i} \leq 0, i=0,1, \cdots, n$.

Theorem. Let $\mathcal{P}$ be a family which satisfies conditions S1 and S2 and is additive. Then $\mathcal{P}$-Ind $X \leq n$ if and only if $X=\bigcup\left\{X_{i}: i=0, \ldots, n\right\}$ with $\mathcal{P}$-Ind $X_{i}$ $\leq 0, i=0, \cdots, n$.

Corollary 2. Let $\mathcal{P}$ be as in the theorem above. Then $\mathcal{P}$-Ind $(A \cup B) \leq$ $\mathcal{P}_{\text {-Ind }} A+\mathcal{P}_{\text {-Ind }} B+1$.

8. The extension theorem. We give here sufficient conditions under which $\mathscr{P}$-Def $=\mathcal{P}$-Ind. $\$ 6$ shows that equality does not hold in general.

8.1. Proposition. Let $\mathcal{P}$ be an additive family. Suppose $\mathcal{P}$ contains the class of topologically complete spaces. If $\mathcal{P}$-Sur $X \leq n$ then in every topologically complete space $Y$ which contains $X$ there is a $\mathcal{P}$-bull $Z$ of $X$ satisfying

(1) $X \subset Z \subset Y$,

(2) Ind $(Z \backslash X) \leq n$. 
Proof. Let $Y$ be topologically complete and $Y \supset X$. There exists a $\mathcal{P}$-kernel $A$ of $X$ such that Ind $X \backslash A \leq n$. By virtue of a theorem of Tumarkin ([11, Theorem II.10]) there exists a $G_{\delta}$ subset $B$ of $Y$ such that $X \backslash A \subset B$ and Ind $B \leq n$. Since $B$ is topologically complete, $B \in \mathcal{P}$. The additivity of $\mathcal{P}$ gives $A \cup B \in \mathcal{P}$. Then $Z=A \cup B$ is a $\mathcal{P}_{\text {-hull }}$ of $X$ contained in $Y$ and Ind $(Z \backslash X) \leq$ Ind $B \leq n$.

The extension theorem. Suppose $\mathcal{P}$ satisfies conditions $\mathrm{S} 1$ and $\mathrm{S} 2$ and is additive. Suppose $\mathcal{P}$ contains the class of topologically complete spaces. Then $\mathcal{P}$-Ind $=\mathcal{P}$-Def.

Proof. Corollary 2 of $\$ 5.1$ and Theorem 4.7 give $\mathcal{P}_{\text {-Ind }} \leq \mathcal{P}_{\text {-Def. The preced- }}$ ing proposition and Theorem 5.1 complete the proof.

The difference theorem. Let $\mathcal{P}$ be as in the extension theorem. Then $\mathcal{P}$ Ind $X \leq n$ if and only if there is a $\mathcal{P}$-bull $Y$ and a $\mathcal{P}$-kernel $Z$ of $X$ with Ind $Y \backslash Z \leq n$.

Proof. Sufficiency follows from Theorem 5.1. To prove the necessity we observe that the extension theorem gives a $\mathcal{P}$-hull $Y$ such that Ind $Y \backslash X \leq n$. We infer from Tumarkin's theorem [11, Theorem II.10] that there is an $F_{\sigma}$ subset $Z$ of $Y$ with $Y \backslash X \subset Y \backslash Z$ and Ind $Y \backslash Z \leq n$. Since $Y \in \mathcal{P}$ and $\mathcal{P}$ is $F_{\sigma}$ monotone the necessity follows.

Example. For every ordinal number $a, 2 \leq a<\Omega$, let $\mathbb{Q}(a)$ denote the family of absolute Borel sets of additive class $a . X$ is said to be an absolute Borel set of additive class $a$ if $X$ is a Borel set of additive class $a$ in every (metric) space $Y$ which contains $X$. It is a classical theorem that $X \in \mathbb{Q}(\alpha)$ if (and only if) $X$ is a Borel set of additive class $\alpha$ in some complete space $Y$ which contains $X$ [7]. It is not hard to show that $\mathscr{Q}(\alpha)$ is additive, satisfies $S 1$ and contains the class of complete spaces. For the nontrivial condition S2 see [3].

\section{REFERENCES}

1. J. M. Aarts, Completeness degree. A generalization of dimension, Fund. Math. 63 (1968), 27-41. MR 38 \#666.

2. - A characterization of strong inductive dimension, Fund. Math. 70 (1971), 147-155.

3. R. Engelking, On Borel sets and B-measurable functions in metric spaces, Prace. Mat. 10 (1967), 145-149. MR 35 \#66.

4. J. de Groot, Topologische Studiën. Compactificatie, Voortzetting van Afbeeldingen en Samenhang, Thesis, University of Groningen, 1942, 102 pp. MR 7, 135.

5. J. de Groot and T. Nishiura, Inductive compactness as a generalization of semicompactness, Fund. Math. 58 (1966), 201-218. MR 33 \#890.

6. W. Hurewicz, Normalbereiche und Dimensionstheorie, Math. Ann. 96 (1927), 736764.

7. C. Kuratowski, Topologie. Vol. I, 4ème éd., Monografie Mat., tom 20, PWN, Warsaw, 1958. MR 19, 873 . 
8. A. Lelek, Dimension and mappings of spaces with finite deficiency, Colloq. Math. 12 (1964), 221-227. MR 31 \#716.

9. K. Morita, On the dimension of normal spaces. II, J. Math. Soc. Japan 2 (1950), 16-33. MR 12, 627.

10. Normal families and dimension theory for metric spaces, Math. Ann. 128 (1954), 350-362. MR 16, 501.

11. J. Nagata, Modern dimension theory, Bibliotheca Math., vol. 6, Interscience, New York, 1965. MR $34 \# 8380$.

12. - Some aspects of extension theory in general topology, Internat. Sympos. on Extension Theory, Berlin, 1967, pp. 157-161.

13. T. Nishiura, Inductive invariants and dimension theory, Fund, Math. 59 (1966), 243-262. MR $34 \# 3541$.

14. Ju. M. Smirnov, Über die Dimension der Adjunkten bei Kompaktifizierungen, Monatsb. Deutsch. Akad. Wiss. Berlin 7 (1965), 230-232. MR 33 \#3266.

15. A. H. Stone, Absolute $F_{\sigma}$ spaces, Proc. Amer. Math. Soc. 13 (1962), 495-499. MR 25\#1535; errata, MR 25 p. 1242.

16. G. T. Whyburn, Analytic topology, Amer. Math. Soc. Colloq. Publ., vol. 28, Amer. Math. Soc., Providence, R. I., 1963. MR 32 \#425.

DEPARTMENT OF MATHEMATICS, DELFT INSTITUTE OF TECHNOLOGY, DELFT, THE NETHERLANDS

DEPARTMENT OF MATHEMATICS, WAYNE STATE UNIVERSITY, DETROIT, MICHIGAN 48202 